\title{
Invasive Micropapillary Carcinoma of Mixed Breast Cancer Metastasizing Faster Into Thyroid: A Rare Case Report
}

\author{
Yupei Yu \\ Central South University \\ Ruifeng Wang \\ Central South University \\ Junqi Deng \\ Central South University \\ Jiayu Zhou \\ Central South University \\ Haiyan Zhou \\ Central South University \\ Junpu Wang ( $\nabla$ wang-jp2013@csu.edu.cn ) \\ Central South University
}

\section{Case Report}

Keywords: tumor-to-tumor metastasis, mixed breast cancer, invasive micropapillary carcinoma, thyroid

Posted Date: June 25th, 2021

DOI: https://doi.org/10.21203/rs.3.rs-646070/v1

License: (c) (i) This work is licensed under a Creative Commons Attribution 4.0 International License. Read Full License 


\section{Abstract}

Background: The incidence of metastatic breast cancer tumors in the thyroid gland is very rare, however, invasive micropapillary carcinoma of breast metastasizes to thyroid gland is rarer. As far as we know, there has never been reported that both invasive ductal carcinoma- no special type (IDC-NST) and invasive micropapillary carcinoma (IMPC) exist in breast whereas only the latter metastasizes into the thyroid gland.

Case presentation: we report a case of a 59-year-old Chinese woman first diagnosed as IDC-NST of breast with involvement of axillary lymph node, grade 2 in 2015 . However, five years later, blood tests revealed increased tumor markers. Multiple solid nodules were found in both side of thyroid gland by imaging. Total thyroidectomy and bilateral cervical lymph nodes dissection were presentation: the findings showed both IDC-NST and IMPC in lymph nodes but only the later in thyroid gland. Immunohistochemically, these tumor cells were positive for ER, PR, GATA3 and Mammaglobin, but negative for TG and TTF-1. Ultimately, the patient was diagnosed as mixed breast cancer with metastatic IMPC in thyroid and radiation therapy was still continued.

Conclusion: Apart from the possibility of metastatic no special type of breast cancer to thyroid tumor, IMPC of breast metastasis must be taken into consideration, especially when the patient has a history of breast cancer and thyroid nodes. Accurate diagnose of metastatic breast carcinoma is vital for precise treatment and can improve the prognosis for patients.

\section{Background}

Primary breast and thyroid tumors are invasive and common disease for women in the World and the increase incidence of both have been well documented in recent years[1, 2]. However, metastatic breast cancer invaded into thyroid gland is a rare event for tumor-to-tumor metastasis is recognized as an uncommon phenomenon[3].The familiar distant sites of secondary relapse of breast neoplasm are the bone, liver, brain and lungs except thyroid[4].

Invasive breast cancer is recently divided into invasive ductal carcinoma - no special type (IDC-NST) and other specific subtypes[5]. IMPC is a subtype of invasive breast cancer, one of peculiar variants of IDCNST, accounting for less than $2 \%$ of all breast cancer types, characterized by small, hollow or morule-like clusters of neoplastic cells without fibrovascular cores and are situated in distinct empty spaces[6, 7]. Local lymph nodes and distant organs are easily involved by IMPC of breast and result in poor prognosis of patients. Interestingly, here we present a peculiar case that shows that thyroid was invaded faster by Primary Invasive Micropapillary Carcinoma (IMPC) of the breast as compared to invasive ductal carcinoma (IDC) of breast.

\section{Case Report}


A 59-year-old woman presented to our hospital with complaint of a lump in the right breast in 2015. Thoroughly clinical examinations were undertaken and the puncture biopsy revealed the cancer was invasive ductal carcinoma, no special type, grade 2. Right modified radical mastectomy was performed and postoperative pathology confirmed the previous diagnosis, with involvement of axillary lymph node. Clinically, the stage was T1N1M0(ER90\%+, PR100\%+, HER2/neu2+). The patient received adjuvant chemotherapy and hormone therapy for 3 years.

In 2018, the patient had a relapse of right chest wall and the mass was removed. She was started on additional radiation therapy for breast cancer. After two years later, blood tests revealed increased carcinoembryonic antigen (CEA) level of $12.2 \mathrm{ng} / \mathrm{ml}$ (reference range: $<5.2 \mathrm{ng} / \mathrm{mL}$ ), CA199 of $42.17 \mathrm{U} / \mathrm{ml}$, CA125of $55.99 \mathrm{U} / \mathrm{ml}$. A positron emission tomography-computed tomography scan (PET-CT) was performed and it showed the elevated uptake in thyroid gland mass (SUV max:6.3)(Fig. 1), multiple cervical lymph nodes (SUV max:4.9), and mediastinal lymph nodes (SUV max:7.5). Ultrasonography of the neck revealed that multiple solid nodules present on both side of the thyroid gland with microcalcifications in the right lobe. Total thyroidectomy and bilateral cervical lymph nodes dissection were done. Pathological biopsy revealed that thyroid gland nodules were inclined to metastatic cancer from breast.

Wax blocks and pathological sections of thyroid gland were sent to our Pathology outpatient department for definite diagnosis. In histopathological examination, there were invasive tumors cells in both thyroid lobes, which were formed by pseudopapillary fragments composed of cell clusters with large granular cytoplasm, lacking fibrovascular cores and floating in clear, empty spaces (Fig. 2a). Immunohistochemical study showed that TG (-), TTF-1(-), GCDFP-15(-), ER (3+,6\%), PR (1+,30\%), GATA3(3+) and Mammaglobin (1+) which clarified the origin of the tumor(Fig. 2b-d). A diagnosis of metastatic micropapillary carcinoma of breast to thyroid was made.

For further treatment, paraffin sections from mastectomy specimen and axillary lymph node were reviewed. Histopathology showed both IDC-NST and IMPC which was misdiagnosed initially(Fig. 3a-b). Immunohistochemical staining revealed that characteristics of epithelial membrane antigen (EMA) expression was reversed polarity and E-cadherin was positive in most of the cell membrane of tumor studies (Fig. 3c-d). Ultimately, the patient was diagnosed as mixed breast cancer (IDC-NST and IMPC) with metastatic thyroid tumor. Radiotherapy was continued and the general situation of the patient is better now as follow-up.

\section{Discussion And Conclusion}

Tumor-to-tumor metastasis is defined as the metastasis of primary neoplasm into at least another site and the host tumor must be the true neoplasm[8]. Thyroid gland serves as recipient to non-thyroid sites metastasis, which is a rare phenomenon and could be explained by fast flow of arterial blood, abundant iodine and oxygen content in the highly vascularized thyroid gland[9, 10]. However, the probability of breast cancer metastasizing into thyroid gland is uncommon that few cases were reported at present in 
total[11-14]. There is only one case reported that IMPC of breast metastasizes into thyroid like this case[11].

Here we present a distinct metastatic breast cancer case which has two components of IDC-NST and IMPC in breast. The patient had a metastasis of IDC-NST and IMPC into lymph nodes, but three years later, only the latter invaded into bilateral thyroid lobes. In spite of immunohistochemistry of IMPC could differentiate from IDC, it was still easily misdiagnosed especially when both of subtypes existed. Although mixed breast cancer had a metastasis in axillary lymph node, the IMPC of breast metastasized in much more direct and immediate ways compared with IDC-NST of breast in this patient. In fact, reports have confirmed that IPMC has a more higher proclivity to lymphovascular invasion, lymph node metastasis in comparison of IDC, which can explain this phenomenon[15]. Much more cases should be reported to verify that IMPC metastasizes into distant organs faster than other subtypes of invasive breast cancer.

In clinical data, it showed that the IMPC is easily mixed with other type of invasive neoplasms, IDC in particular. Pure component of IMPC(more than 75\%)is extremely rare[16]. In conclusion, apart from the possibility of metastatic no special type of breast cancer to thyroid tumor, IMPC of breast metastasis must be taken into consideration, especially when the patient has a history of breast cancer and thyroid nodes. It is very important to make an accurate diagnose for IMPC in primary breast cancers to promote therapy and improve prognosis of patients. Specific morphology, immunohistochemical characteristic and gene detection are helpful to diagnose IMPC of breast to thyroid tumor metastasis.

\section{Abbreviations}

IDC-NST: invasive ductal carcinoma-no special type; IDC: invasive ductal carcinoma; IMPC: invasive micropapillary carcinoma; CEA: carcinoembryonic antigen; PET-CT: positron emission tomographycomputed tomography scan; EMA: epithelial membrane antigen.

\section{Declarations}

\section{Acknowledgments}

We thank the Department of Pathology, Xiangya Hospital, Central South University, Hunan, China for assistance with pathological diagnosis.

\section{Authors' contributions}

YYP collected the clinical data and drafted the manuscript. ZHY and WJP made the pathological diagnosis and revised the manuscript. ZJY, WRF and DJQ, offered assistance in image selection. All authors read and approved the final manuscript.

\section{Funding}


Not applicable.

\section{Availability of data and materials}

All data generated or analyzed during this case are included within the article.

\section{Ethics approval and consent to participate}

This case study was approved by the Institutional Ethics Committee of Xiangya Hospital Central South University, Hunan Province, China.

\section{Consent for publication}

1. Written informed consent was obtained from the patient for the publication of this case report and any accompanying images. A copy of the consent form is available for review by the Editor of Diagnostic Pathology.

\section{Competing interests}

The authors declare that they have no competing interests.

\section{References}

1. Roman BR, Randolph GW, Kamani D. Conventional Thyroidectomy in the Treatment of Primary Thyroid Cancer. Endocrinol Metab Clin North Am. 2019;48(1):125-41.

2. Ferlay J, Colombet M, Soerjomataram I, Parkin DM, Pineros M, Znaor A, Bray F. Cancer statistics for the year 2020: an overview. Int J Cancer 2021.

3. Tan W, Tao L, Zhou Z, Yin W, Chen Y. Tumor-to-tumor metastasis: a rare case of breast carcinoma metastasizing to a pheochromocytoma, and a literature review. Diagn Pathol. 2019;14(1):46.

4. Kennecke H, Yerushalmi R, Woods R, Cheang MC, Voduc D, Speers CH, Nielsen TO, Gelmon K. Metastatic behavior of breast cancer subtypes. J Clin Oncol. 2010;28(20):3271-7.

5. Kaya C, Ucak R, Bozkurt E, Omeroglu S, Kartal K, Yazici P, Idiz UO, Mihmanli M. The Impact of Micropapillary Component Ratio on the Prognosis of Patients With Invasive Micropapillary Breast Carcinoma. J Invest Surg. 2020;33(1):31-9.

6. Sinn HP, Kreipe H: A Brief Overview of the WHO Classification of Breast Tumors, 4th Edition, Focusing on Issues and Updates from the 3rd Edition. Breast Care (Basel) 2013, 8(2):149-154.

7. Shi WB, Yang LJ, Hu X, Zhou J, Zhang Q, Shao ZM. Clinico-pathological features and prognosis of invasive micropapillary carcinoma compared to invasive ductal carcinoma: a population-based study from China. PLoS One. 2014;9(6):e101390.

8. Sayegh ET, Henderson GA, Burch EA, Reis GF, Cha S, Oh T, Bloch O, Parsa AT. Intrameningioma metastasis of breast carcinoma. Rare Tumors. 2014;6(2):5313. 
9. Hegerova L, Griebeler ML, Reynolds JP, Henry MR, Gharib H. Metastasis to the thyroid gland: report of a large series from the Mayo Clinic. Am J Clin Oncol. 2015;38(4):338-42.

10. Yoon JH, Kim EK, Kwak JY, Moon HJ, Kim GR. Sonographic features and ultrasonography-guided fine-needle aspiration of metastases to the thyroid gland. Ultrasonography. 2014;33(1):40-8.

11. Kiziltan G, Bozdogan N, Ozaslan C. Breast cancer metastasis into thyroid papillary carcinoma: A case report. The Breast Journal 2021.

12. Raveendrannair AK, Mathews A, Varghese BT, Jayasree K. Papillary carcinoma thyroid serving as recipient tumor to carcinoma breast: A rare example of tumor-to-tumor metastasis. Indian J Pathol Microbiol. 2019;62(1):122-4.

13. Zhou L, Chen L, Xu D, Shao Q, Guo Z, Ge M. Breast cancer metastasis to thyroid: a retrospective analysis. Afr Health Sci. 2017;17(4):1035-43.

14. Plonczak AM, DiMarco AN, Dina R, Gujral DM, Palazzo FF. Breast cancer metastases to the thyroid gland - an uncommon sentinel for diffuse metastatic disease: a case report and review of the literature. J Med Case Rep. 2017;11(1):269.

15. De la Cruz C, Moriya T, Endoh M, Watanabe M, Takeyama J, Yang M, Oguma M, Sakamoto K, Suzuki $\mathrm{T}$, Hirakawa $\mathrm{H}$, et al. Invasive micropapillary carcinoma of the breast: clinicopathological and immunohistochemical study. Pathol Int. 2004;54(2):90-6.

16. Amendoeira I, Magalhães J, Damasceno M. Invasive micropapillary carcinoma of the breast: are the pure forms more aggressive than the mixed forms? The breast journal. 2003;9(4):337-8.

\section{Figures}




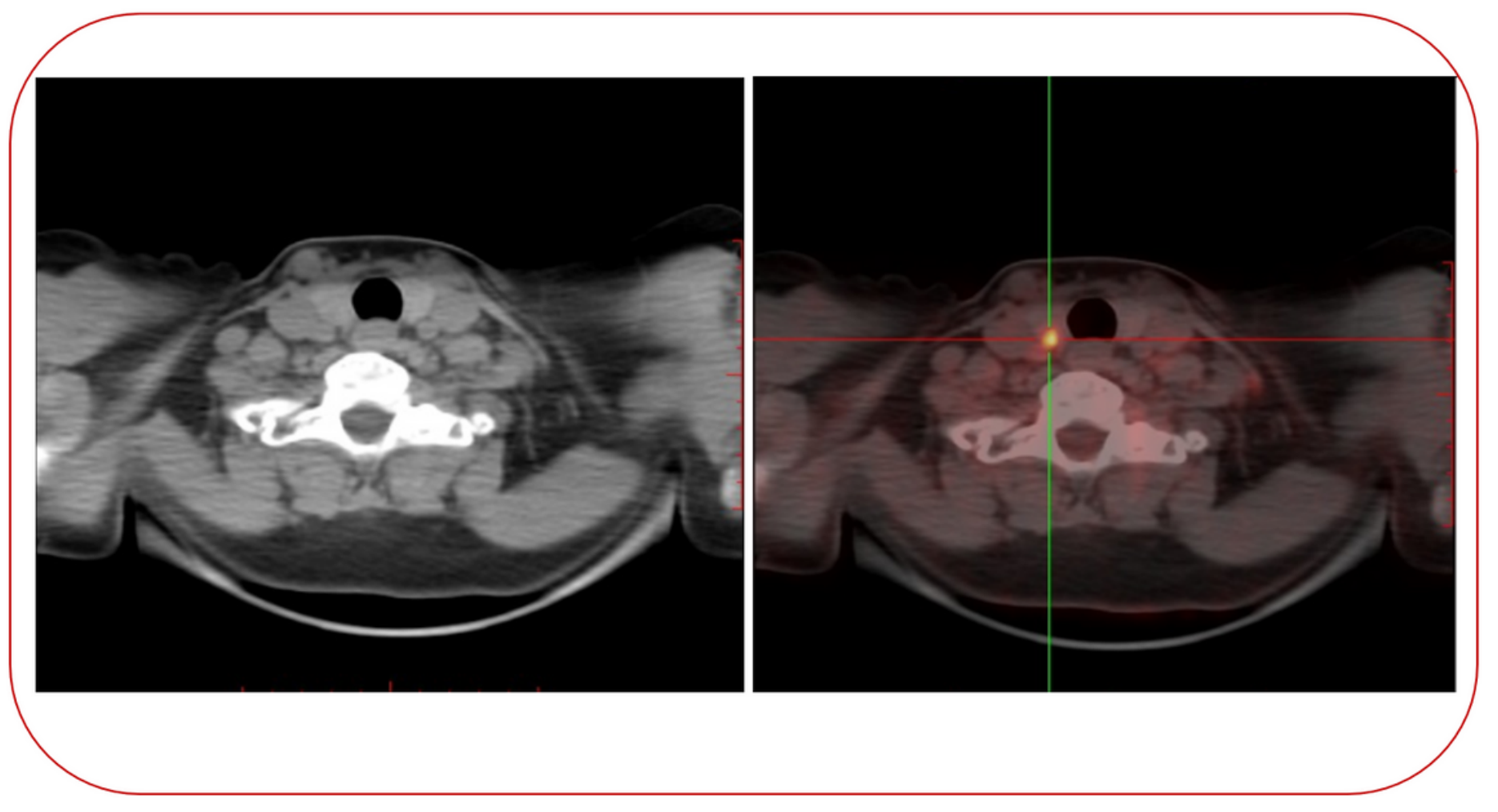

Figure 1

PET-CT revealed increased focal FDG uptake in the thyroid gland mass. 


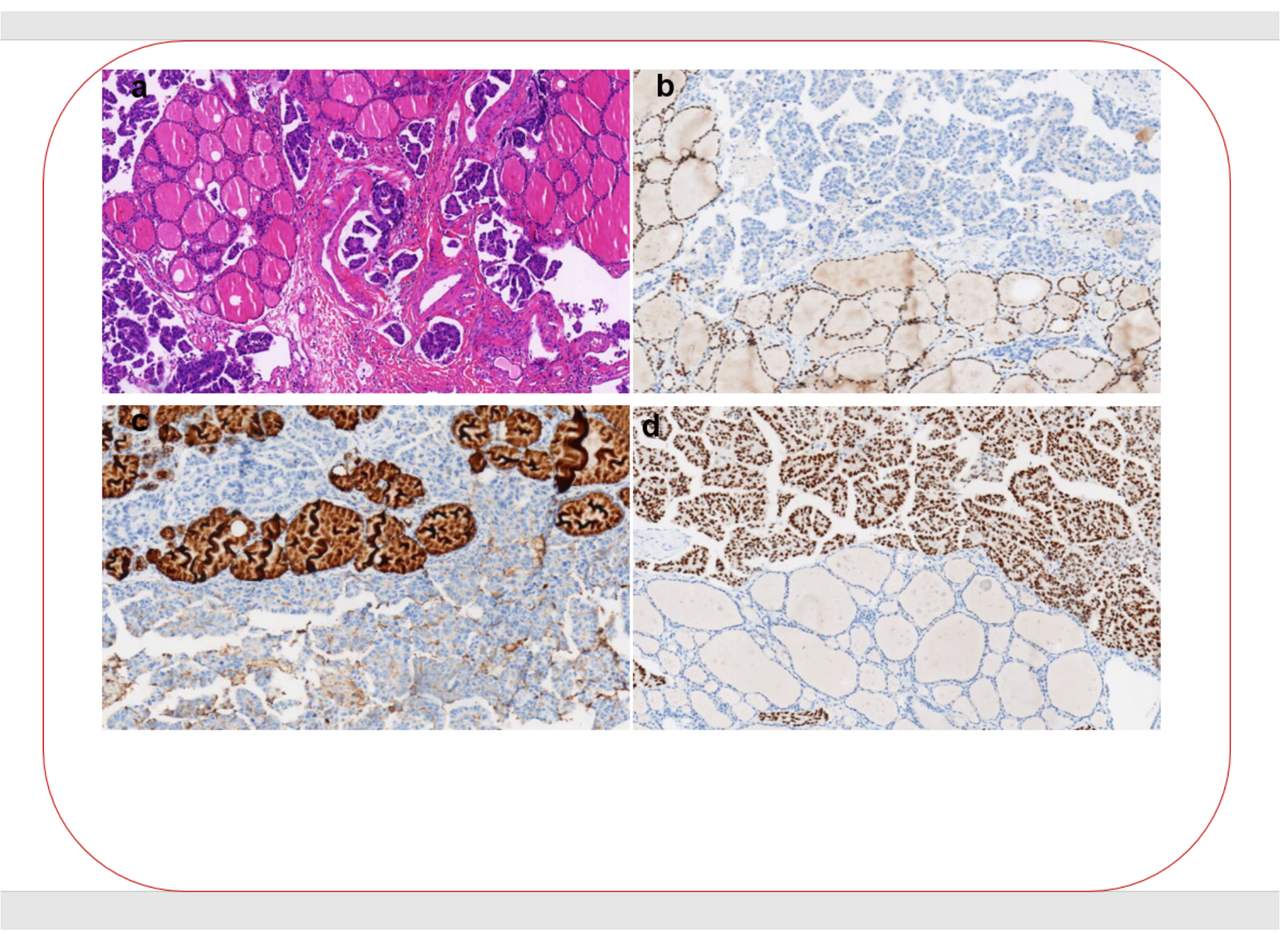

\section{Figure 2}

a. Metastatic invasive micropapillary carcinoma in the thyroid gland; $b$. thyroid transcription factor-1(TTF-1) negativity in the metastatic tumor area; $c$. Thyroglobulin negativity in the breast cancer; d. GATA-3 positivity in breast cancer metastasis cells. (a\&b\&c\&d: magnifation $\times 40$ ) 

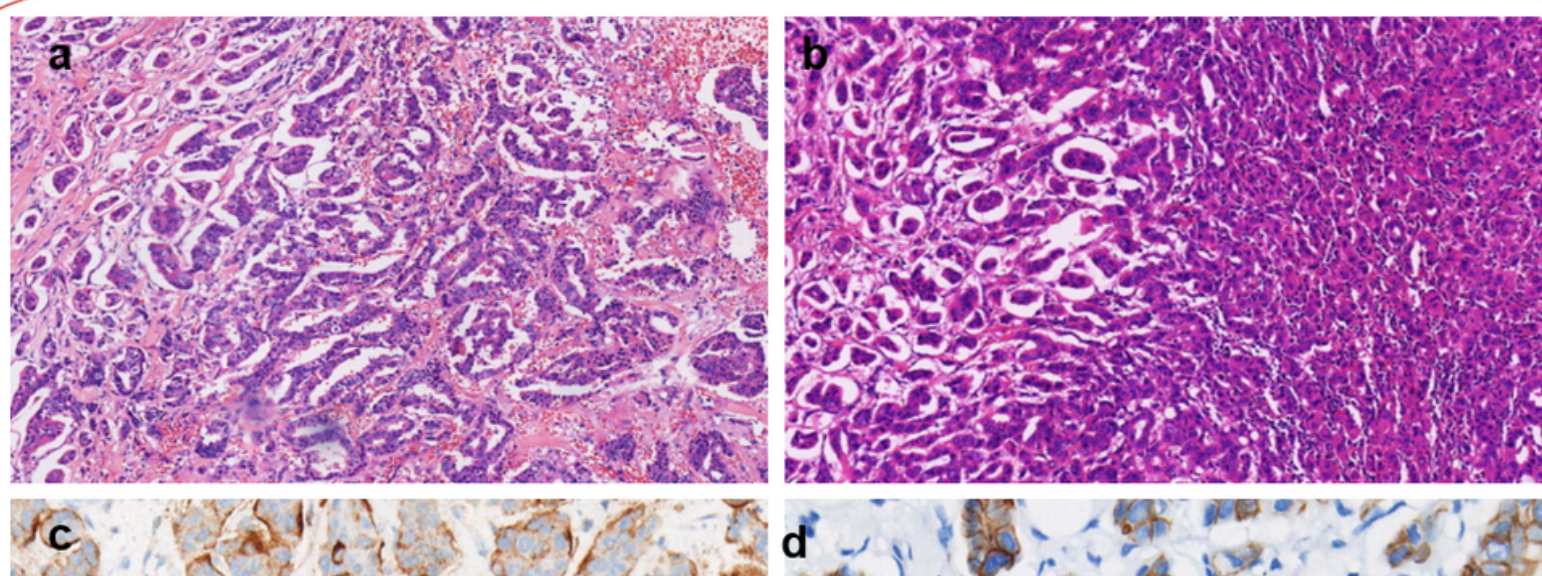

c

is

to
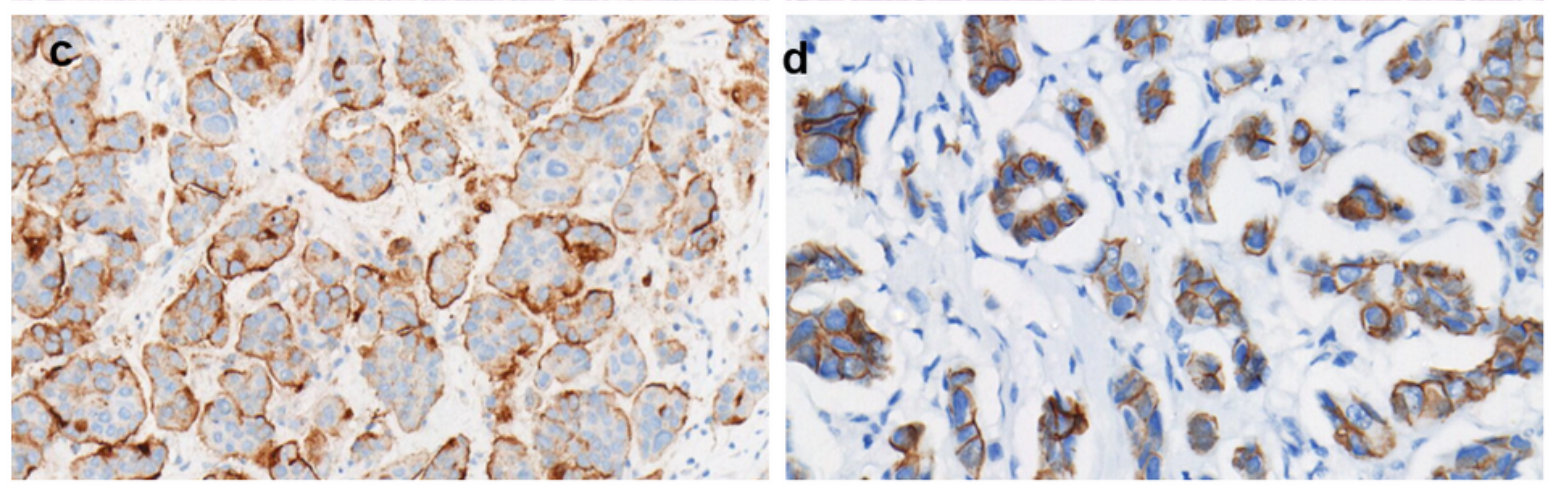

\section{Figure 3}

a\&b. both invasive micropapillary carcinoma and invasive ductal carcinoma in breast and axillary lymph node (a\&b: magnifation $\times 40$ ); c. characteristics of EMA expression showed reversed polarity of IMPC cells in axillary lymph node(magnifation $\times 100)$; $d$. E-cadherin was positive in most of the cell membrane of tumor cells in axillary lymph node (magnifation×200). 